\title{
Editorial
}

\section{Acknowledgment to Reviewers of Languages in 2021}

\section{Languages Editorial Office}

MDPI AG, St. Alban-Anlage 66, 4052 Basel, Switzerland; languages@mdpi.com

Rigorous peer-reviews are the basis of high-quality academic publishing. Thanks to the great efforts of our reviewers, Languages was able to maintain its standards for the high quality of its published papers. Thanks to the contribution of our reviewers, in 2021, the median time to first decision was 35 days and the median time to publication was 94 days. The editors would like to extend their gratitude and recognition to the following reviewers for their precious time and dedication, regardless of whether the papers they reviewed were finally published:

Agathopoulou, Eleni

Agustin Llach, Maria Pilar

Ahern, Aoife Kathleen

Alalou, Ali

Alba De La Fuente, Anahi

Alexiadou, Artemis

Allen, Todd James

Al-Tairi, Hamed

Álvarez De La Fuente, Esther

Anastasio, Simona

Andersson, Annika

Andorno, Cecilia

Archibald, John

Armstrong, Timothy Currie

Arnhold, Anja

Arslangul, Arnaud

Asadi, Homa

Astori, Davide

Babatsouli, Elena

Bagna, Carla

Baird, Brandon O.

Ballinger, Susan

Balogh, András

Balteiro, Isabel

Bar-Moshe, Assaf

Beaudrie, Sara

Bednall, James

Belk, Zoë

Bell, Elise

Bellik, Jennifer

Bello-Uriarte, Adrián

Benítez, Montserrat

Benkato, Adam

Berger, Armin

Blackledge, Adrian
Blum, Susan

Bobkina, Jelena

Bóna, Judit

Bonar, Gary

Bonaventura, Patrizia

Booth, Hannah

Borger, Linda

Borreguero Zuloaga, Margarita Natalia

Bove, Kate

Bowler, Margit

Bowles, Melissa

Breitbarth, Anne

Brekelmans, Gwen

Brown, Joshua

Brustad, Kristen

Budach, Gabriele

Bueno-Alastuey, M. Camino

Burgo, Clara

Busse, Vera

Butt, Miriam

Cabal-Jiménez, Munia

Campos-Dintrans, Gonzalo

Carvalho, Janayna

Castek, Jill M

Celata, Chiara

Celaya, M. Luz

Chambless, Della

Champoux-Larsson, Marie-France

Cho, Grace

Chung, Siaw-Fong

Cocchi, Gloria

Cognola, Federica

Comajoan, Llorenç

Coutsougera, Photini

Crasborn, Onno 
Cripps, Jody H.

Crocco, Claudia

Cruz Enríquez, Maura

Cruz-Santos, Anabela

Cui, Aletheia

Custodio, Brenda

Czypionka, Anna

Da Silva, Emanuel Angelo

Dam, Lotte

Dancygier, Barbara

D'Anna, Luca

Darquennes, Jeroen

Das, Sonia N.

Daugaard, Line Møller

Davidson, Justin

Dawson, Virginia

De Souza, Ricardo Napoleão

Defina, Rebecca

De-la-Mota, Carme

Delgado, Rodrigo

Den Dikken, Marcel

Deschrijver, Cedric

Despoina, Papadopoulou

Ducasse, Anamaria

Duek, Susanne

Edmonds, Amanda

Egan, Patricia

Eibensteiner, Lukas

Embarki, Mohamed

Enns, Charlotte

Erhart, Pascale

Fafulas, Stephen

Fagyal, Zsuzsanna

Fairclough, Marta

Falk, Ylva

Farasyn, Melissa

Farwaneh, Samira

Fernández Cuenca, Sara

Fernández-Mallat, Víctor

Fernández-Sánchez, Javier

Filipe, Marisa

Foolen, Ad

Forsberg Lundell, Fanny

Fortune, Tara

Fougeron, Cécile

Friedman, Debra

Friesen, Deanna

Frolova, Anna

Gangopadhyay, Ishanti

García-del-Real, Isabel

Gasser, Emily A.
Georgiou, Georgios P.

Gerstenberg, Anette

Giannikas, Christina Nicole

Giraudo, Hélène

Giudici, Alberto

Gobes, Sharon M. H.

Gogonas, Nikolaos

Goldin, Michele

Gomashie, Grace A.

Gonzalez, Becky Halloran

González, Carolina

Good, Jeff

Gradoville, Michael

Greenspon, Emma B.

Grichkovtsova, Ioulia

Grondelaers, Stefan

Grubic, Mira

Guajardo, Gustavo

Guardiano, Cristina

Guekguezian, Peter Ara

Guerrero, Jairo

Gupton, Timothy

Gut, Ulrike

Gutiérrez Pérez, Regina

Guy, Gregory

Guzzo, Natália Brambatti

Hadermann, Pascale

Hadodo, Matthew John

Hallett, Jennifer S.

Hamlaoui, Fatima

Hammond, Mike

Hartig, Alissa

Heil, Jeanne

Hellekjær, Glenn Ole

Heredia Mantis, María

Hernández, Todd

Hicks, Troy

Hill, Virginia

Hinton, Leanne

Hornsby, Michael

Hos, Rabia

Howerton-Fox, Amanda

Hurtado, Luz Marcela

Hussain, Qandeel

Irimia, Monica

Ito, Aine

Ivan, Loredana

Ivanova, Olga

Jaber, Aziz

Jacobsen, Jannes

Jessner-Schmid, Ulrike 
Johnston, Suzanne

Juvonen, Päivi

Kacetl, Jaroslav

Kapatsinski, Vsevolod

Kapitonov, Ivan

Karatsareas, Petros

Karlsson, Fredrik

Karras, Ioannis

Kastner, Itamar

Kelly, Debra

Kempas, Ilpo

Khomchenkova, Irina

Kiliańska-Przybyło, Grażyna

Knipe, John

Koch, Harold

Köpke, Barbara

Kornder, Lisa

Kornfilt, Jaklin

Koulidobrova, Elena

Krapova, Iliyana

La Rosa, Cristina

Lai, Rosangela

Laihonen, Petteri

Laleko, Oksana

Laughren, Mary

Leddy-Cecere, Thomas

Lee, Chia-Cheng

Lee, Jiyoon

Limerick, Philip P.

Lindqvist, Christina

Lipkind, Dina

Lipski, John

Litty, Samantha

Llinàs-Grau, Mireia

Llop, Ares

Llopart-saumell, Elisabet

Llopis Cardona, Ana

Lloyd-Smith, Anika

Loester, Barbara

Longhi, Julien

López, Luis

Loureiro-Rodríguez, Verónica

Loza, Sergio

Lozano Argüelles, Cristina

Lucas, Christopher

Luchkina, Tatiana

Lukin, Annabelle

Luque, Alicia

Mac Giolla Chriost, Diarmait

Magidow, Alexander

Maligkoudi, Christina
Manley, Marilyn

Mannheim, Bruce

Mansfield, John

Mantell, James T.

Manzini, Maria Rita

Markey, Michael

Martel-Sauvageau, Vincent

Marten, Heiko F.

Martínez Lirola, María

May, Stephen

Mckee, Rachel

Meakins, Felicity

Mendoza, Claudia Holguín

Mengozzi, Alessandro

Mereu, Daniela

Michot, Marie-Eve

Mifka-Profozic, Nadia

Mikulski, Ariana

Miller, Catherine

Mion, Giuliano

Mitrofanova, Natalia

Mouti, Anna

Moyer, Alene

Mücke, Doris

Mullan, Kerry

Mushin, Ilana

Nagano, Tomonori

Nagaya, Naonori

Nagy, Naomi

Nakao, Shuichiro

Naranjo, Matías Guzmán

Natvig, David

Nedashkivska, Alla

Nguyen, Mai

Ní Dhiorbháin, Aisling

Nicoladis, Elena

Nicolae, Alexandru

Nicolarakis, Onudeah D.

Nicolás, Estrella

Oittinen, Tuire

Ojea, Ana

Olson, Daniel J.

Ono, Yoshi

Orozco, Rafael

Osborne, Denise

Osborne, Sam

Otto, Ana

Palacios, Ignacio Martínez

Paoli, Sandra

Papachristou, Vicky

Parafita Couto, M Carmen 
Park, Seong Man

Parra, Maria

Patekar, Jakob

Patil, Umesh

Patin, Cédric

Pawlak, Mirosław

Pensalfini, Rob

Perez-Cortes, Silvia

Perono Cacciafoco, Francesco

Perry, Conrad

Phyak, Prem

Pierce, Marc

Pino, Aymé

Pisabarro Sarrió, Silvia

Pischedda, Pier

Ponsonnet, Maia

Pontikas, George

Pope, Joshua

Posio, Pekka

Prochazka, Stephan

Puigdevall Serralvo, Maite

Pujolar, Joan

Purnell, Thomas C.

Putnam, Michael

Rafat, Yasaman

Raimy, Eric

Ransmayr, Jutta

Rato, Anabela

Reagan, Timothy

Reed, Lisa

Reed, Paul E.

Regueira Fernández, Xosé-Luis

Relyea, Jackie Eunjung

Repiso, Isabel

Requena, Pablo E.

Rice, Keren D.

Ritt-Benmimoun, Veronika

Ritz, Marie-Eve

Roberts, Ian

Rodriguez Padial, Ana

Rodriguez, Estrella

Rojas, Sandra

Rose, Françoise

Rosenthall, Samuel

Rosseel, Laura

Rossi, Olena

Rubio, Fernando

Salaberry, M. Rafael

Salmons, Joseph

Sánchez, Liliana

Sanfelici, Emanuela
Santos Marinas, Enrique

Sanz-Sánchez, Israel

Sarvasy, Hannah

Schmid, Monika

Schönström, Krister

Schroeder, Christoph

Schultze-Berndt, Eva

Schwartz, Geoffrey

Shimanskaya, Elena

Shively, Rachel

$\mathrm{Si}$, Aung

Silber-Varod, Vered

Sleeman, Petra

Smith, Hilary

Smith, Laura

Sonnenschein, Aaron Huey

Souag, Lameen

Speyer, Augustin

Spina, Stefania

Spotti, Max

Stefanich, Sara

Steinkrauss, Rasmus

Stoehr, Antje

Stuart, Nichola

Subasi, Seyda

Sudhoff, Stefan

Sunderman, Gretchen

Tabain, Marija

Tagliamonte, Sali A.

Taine-Cheikh, Catherine

Tallman, Adam James Ross

Tanaka, Hiromasa

Tarpomanova, Ekaterina

Tejedo-Herrero, Fernando

Terry, Kristen

Thordardottir, Elin

Thurgood, Graham

Topping, Keith

Torgersen, Eivind Nessa

Torres-Tamarit, Francesc

Tosco, Mauro

Tragant, Elsa

Trommer, Jochen

Tse, Holman

Tsiplakou, Stavroula

Turner, Mike

Uffmann, Christian

Usanova, Irina

Vágvölgyi, Réka

Valdivia, Victor

Van De Craen, Piet 
Van Putten, Marijn

Van Steensel, Roel

Vandekerckhove, Reinhild

Vanden Bosch Der Nederlanden, C.M.

Vanden Wyngaerd, Emma

Vargas-Sierra, Chelo

Vázquez Rozas, Victoria

Vihman, Virve-Anneli

Vogt, Karin
Walkden, George

Watson, Janet C.E.

Weiss, Helmut

Werth, Alexander

Zabrodskaja, Anastassia

Zaenen, Annie

Zárate-Sández, Germán

Zas Varela, Luz

Zhou, Xiaodi 